\title{
1 A boundary error sensing arrangement for virtual sound barriers to reduce noise radiation through openings
}

$6 \quad{ }^{1}$ Centre for Audio, Acoustics and Vibration, Faculty of Engineering and IT, University of Technology Sydney, NSW 2007, Australia

$8 \quad{ }^{2}$ Key Laboratory of Modern Acoustics and Institute of Acoustics, Nanjing University,

$10{ }^{3}$ Department of Mechanical Engineering, The University of Western Australia, WA 6009, 


\section{ABSTRACT}

20 Previous work has demonstrated that sound radiation through a cavity opening can be

21 reduced with secondary sources at the edge of the opening, but the error microphones are

22 implemented over the entire opening, which might affect the natural ventilation, lighting,

23 and especially the access through the opening in some applications. A boundary error

24 sensing arrangement is proposed and investigated in this paper. It is found that a

25 double-layer error microphone arrangement achieves better performance than a

26 single-layer one. Although its performance is not as good as the arrangement with error

27 microphones distributed over the entire opening, it is preferable in some applications

28 because it does not block the opening. It is also found that there exists an upper-limit

29 frequency for the systems with error microphones installed at the edge, which is related

30 to the size of the opening and can be increased by adding more layers of error

31 microphones at the edge. This work demonstrates the possibility of developing an almost

32 invisible virtual sound barrier system that can block sound transmission through an

33 opening without affecting its functionalities.

35 PACS numbers: 43.50.Ki 


\section{I. INTRODUCTION}

40 Openings are important for lighting, natural ventilation, and access through

41 buildings and enclosures; however, they introduce sound transmission paths that reduce

42 the transmission loss of the whole structures. Traditional passive noise control methods,

43 such as applying porous materials, micro-perforated absorbers, and quarter-wave

44 resonators, require that the opening be sealed and/or filled with these materials or

45 structures, so they are inappropriate for some applications. ${ }^{1-3}$ Compared with passive

46 noise control, active noise control (ANC) can maintain the functionalities of the openings

47 and works effectively, especially for low-frequency noise.

48 Using Huygens' principle as the theoretical basis, it has been demonstrated in

49 previous work that sound power radiation through openings to the outside can be

50 effectively reduced by placing a sufficient number of secondary sources over the entire

51 opening. ${ }^{4-8}$ To avoid putting secondary sources in the middle of the opening, a

52 double-layer secondary source system at the edge of the opening has been proposed and

53 both the numerical simulation and experiment results demonstrate the feasibility of this

54 configuration. ${ }^{9}$ Due to reciprocity, applying secondary sources only on the frame of the

55 opening can also reduce sound radiation through the opening to inside the cavity. ${ }^{10}$

56 However, in these systems, error microphones are located over the entire opening, which

57 might affect access through the opening.

58 To achieve global sound power reduction, error microphones should provide 
59 information that is proportional to the sound power of the system. The sound power can

60 be measured according to ISO 3744 with ten or twenty microphones on a hemisphere. ${ }^{11-12}$

61 The hemisphere's radius should be larger than each of the three values: twice the largest

62 source dimension, a quarter of the wavelength of interest, and $1 \mathrm{~m} .{ }^{11}$ Therefore, it is not

63 practical to apply error microphones at these locations in some applications, especially

64 for a large noise source in some applications where a compact system is desired. Since

65 sound power is the integral of sound intensity over a surface around the noise source,

66 Berry et al. used the near-field sound intensity as the cost function, but finds that due to

67 its signed quantity, there are difficulties associated with sound intensity minimization. ${ }^{13}$

68 In order to achieve effective global control with error microphones in the near field,

69 their positions need to be optimized. The optimal positions for error microphones are the

70 locations where noise reduction is the greatest when minimizing the total radiated sound

71 power. ${ }^{14}$ Shafer et al. demonstrated experimentally that the measured near-field sound

72 pressure map approximates the one when minimizing the sound power if the error

73 microphones are at these ideal positions and that moving them to other locations will

74 greatly deteriorate the sound power reduction performance. ${ }^{15}$

75 There has been much work reported on optimizing the positions of error

76 microphones. For simple cases like using a single-channel ANC system to reduce the

77 primary noise generated by a monopole or dipole, the optimal positions can be

78 investigated theoretically. ${ }^{16}$ If the primary sound field is complicated, genetic algorithms 
79 and simulated annealing algorithms can be used to search for the optimal positions of

80 error microphones, but it is usually difficult to obtain the global optimal solution and the

81 optimal solutions might be different for noise at different frequencies. ${ }^{17-19}$

82 Virtual sensing is another way to achieve effective noise control with error

83 microphones in the near field. In this strategy, physical error microphones near the

84 primary source are used to estimate the sound pressures at virtual error sensor locations in

85 the far field for minimization. ${ }^{20}$ If the virtual error sensors are at the locations defined in

86 ISO 3744, the sound power of the system can be minimized. However, most previous

87 work on virtual sensing focuses on local control, and its feasibility to achieve global

88 sound power reduction remains to be investigated. ${ }^{21-23}$ Another problem with the virtual

89 sensing approach is that it requires preliminary identification of the system.

90 In this paper, a simple configuration of error microphones is proposed that installs

91 error microphones at the edge of the cavity opening. The performances of single-layer

92 and double-layer error microphones at the edge are compared. The upper-limit frequency

93 of effective noise control for such a boundary error microphone arrangement and its

94 relationship with the opening size are explored.

\section{II. THEORY}

96 Schematic diagrams of the single-layer and double-layer error microphone

97 arrangements are shown in Fig. 1. In the single-layer system, the error microphones are

98 distributed along the edge of the opening. In the double-layer system, two layers of error 
99 microphones are installed at two different heights along the edge, and they have the same

$100 x-y$ coordinates. All the five walls of the cavity are rigid, so sound outside the cavity is

101 solely that transmitted through the opening. The primary noise source is assumed to be a

102 monopole point source inside the cavity.

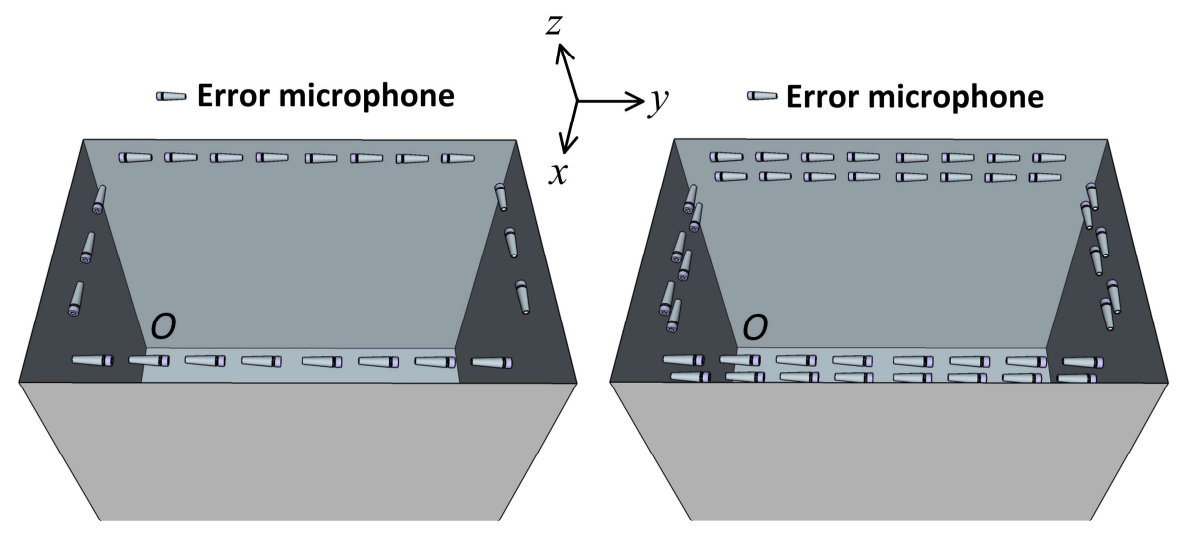

103

(a)

(b)

104 FIG. 1. (Color online) Schematic diagrams of (a) single-layer error microphones at the edge and (b) double-layer error microphones at the edge.

The sum of the squared sound pressures at all the error microphones with a control

108 effort constraint is defined as the cost function ${ }^{4}$

$$
J=\mathbf{p}^{\mathrm{H}} \mathbf{p}+\beta \mathbf{q}_{\mathrm{s}}^{\mathrm{H}} \mathbf{q}_{\mathrm{s}},
$$

110 where $\mathbf{p}$ is the vector of sound pressures at the error points, $\mathbf{q}_{\mathrm{s}}$ is the vector of the

111 strengths of secondary sources, and $\beta$ is a real number to constrain the outputs of

112 secondary sources. ${ }^{24}$ After minimizing Eq. (1), the optimized strengths of the secondary

113 sources can be obtained with 


$$
\mathbf{q}_{\mathrm{s}}=-\left(\mathbf{Z}_{\mathrm{se}}^{\mathrm{H}} \mathbf{Z}_{\mathrm{se}}+\beta \mathbf{I}\right)^{-1} \mathbf{Z}_{\mathrm{se}}^{\mathrm{H}} \mathbf{Z}_{\mathrm{pe}} q_{\mathrm{p}},
$$

115 where $\mathbf{Z}_{\mathrm{se}}$ is the acoustic transfer function matrix between the secondary sources and the

116 error microphones, $\mathbf{Z}_{\mathrm{pe}}$ is the acoustic transfer function vector from the primary source to

117 the error microphones, and $q_{\mathrm{p}}$ is the strength of the primary source.

118 The noise reduction of the system is defined as the difference between the sound

119 power levels of the system with and without control

$$
\mathrm{NR}=10 \log _{10} \frac{W_{\text {off }}}{W_{\text {on }}}
$$

121 where $W_{\text {off }}$ and $W_{\text {on }}$ are the sound powers of the system without and with control,

122 respectively. The sound power $W_{\text {off }}$ can be calculated as the integral of sound intensity

123 over the opening area $S$

$$
W_{\text {off }}=\frac{1}{2} \iint_{S} \operatorname{Re}\left\{p_{\mathrm{po}}^{*} v_{\mathrm{po}}\right\} \mathrm{d} S,
$$

125 where $p_{\mathrm{po}}$ and $v_{\mathrm{po}}$ are the sound pressure and normal particle velocity generated by the

126 primary source at the opening. The sound power $W_{\text {on }}$ is the sum of the contributions of

127 the primary source and all the secondary sources

$$
W_{\text {on }}=\frac{1}{2} \iint_{S} \operatorname{Re}\left\{\left[p_{\mathrm{po}}+p_{\mathrm{so}}\right]^{*}\left[v_{\mathrm{po}}+v_{\mathrm{so}}\right]\right\} \mathrm{d} S .
$$

129 In Eq. (5), $p_{\mathrm{so}}$ and $v_{\mathrm{so}}$ are the sound pressure and normal particle velocity generated by the

130 secondary sources with the optimized strengths $\mathbf{q}_{\mathrm{s}}$, which are calculated with Eq. (2).

\section{III. SIMULATIONS AND DISCUSSIONS}




\section{A. Comparison between single-layer and double-layer error microphones at the edge}

133 In the simulations, the dimensions of the open cavity are $0.3 \mathrm{~m} \times 1.0 \mathrm{~m} \times 0.598 \mathrm{~m}\left(l_{x}\right.$

$134 \times l_{y} \times l_{z}$ ), and the size of the opening is $0.3 \mathrm{~m} \times 1.0 \mathrm{~m}$. The modal superposition method

135 in Ref. [8] is applied to obtain the theoretical acoustic transfer functions and the sound

136 pressure and particle velocity at the opening to calculate the sound power of the system.

137 The primary source is a monopole point source at $(0.01,0.01,0.01) \mathrm{m}$ with a strength of

$138 q_{\mathrm{p}}=2 \times 10^{-4} \mathrm{~m}^{3} / \mathrm{s}$. The secondary sources are also monopole point sources, and forty-four

139 of them are evenly distributed at the height of $z=0.448 \mathrm{~m}$.

140 Numerical simulations show that the number of error microphones in single-layer

141 and double-layer systems does not significantly affect the noise reduction performance if

142 the number of error microphones is larger than that of secondary sources to prevent the

143 system from being underdetermined, so more error microphones than secondary sources

144 are used in the simulations. A total of 56 error microphones in the single-layer and

145 double-layer systems are applied at the opening, and their positions are shown in Figs.

146 2(a) and (b). The results for the traditional arrangement of evenly distributed error

147 microphones, shown in Fig. 2(c), are also given for comparison. The error microphones

148 in the single-layer and evenly distributed systems are at the height of $z=0.588 \mathrm{~m}$, and

149 those in the double-layer system are at $z=0.568 \mathrm{~m}$ and $z=0.588 \mathrm{~m}$ planes. 


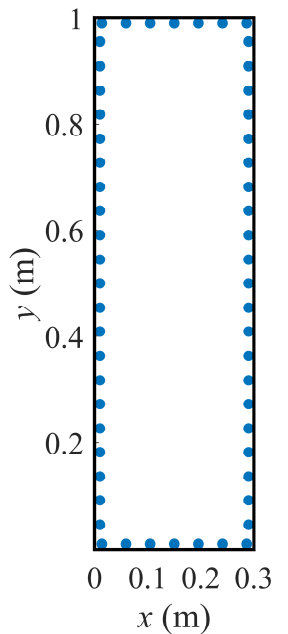

(a)

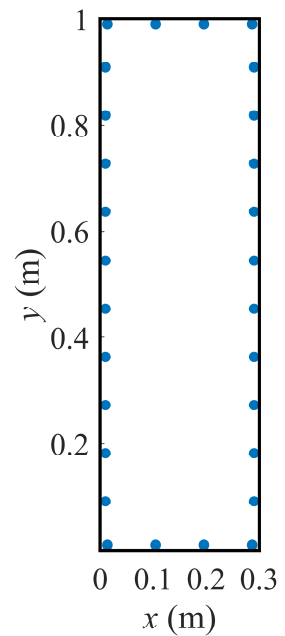

(b)

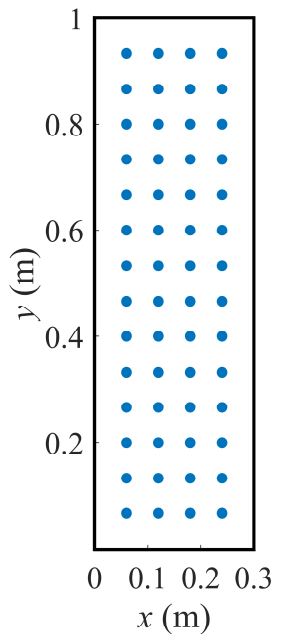

(c)

151 FIG. 2. (Color online) The positions of error microphones in the $x-y$ plane: (a)

152 single-layer error microphones at the edge, (b) one of the layers of the double-layer error

153 microphones at the edge, and (c) evenly distributed error microphones.

155 The sound power levels of the system with and without ANC are shown in Fig. 3.

156 The theoretically best noise reduction performance obtained by minimizing the sound

157 power is also included for comparison. ${ }^{24}$ It can be seen that the evenly distributed error

158 microphones achieve the highest noise reduction, and that the double-layer error

159 microphones perform better than the single-layer ones. Taking $1000 \mathrm{~Hz}$ as an example,

160 the noise reduction achieved with the single-layer error microphones is $14.4 \mathrm{~dB}$ while

161 that with the double-layer ones is $40.5 \mathrm{~dB}$. 


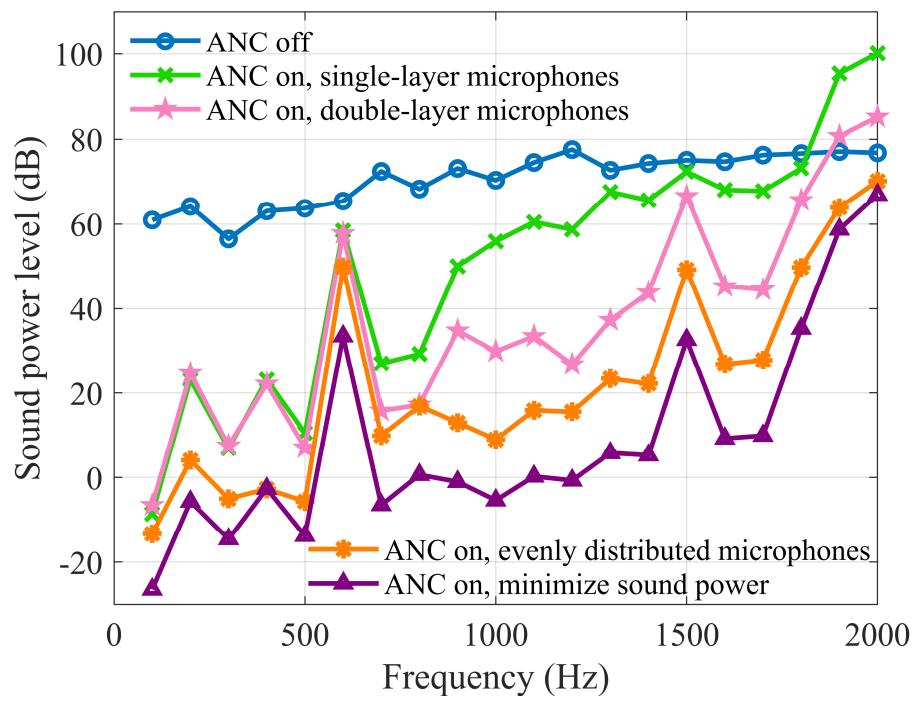

163 FIG. 3. (Color online) Sound power levels with and without ANC under different

164 configurations of error microphones compared with the theoretically maximum noise

reduction (minimize sound power).

167 The spatial distributions of the sound power level and the decibel level of the normal

168 particle velocity at the opening with and without ANC are shown in Fig. 4. It can be seen

169 that the effective noise reduction zones are limited with the single-layer error

170 microphones, which are located around the edge of the opening; however, the noise

171 reduction zones are significantly enlarged with the double-layer ones. Both the sound

172 pressure and normal particle velocity can be significantly reduced after control with the

173 double-layer error microphones, which is similar to the result when using acoustic energy

174 density as the cost function to reduce noise in enclosures. ${ }^{25}$ 


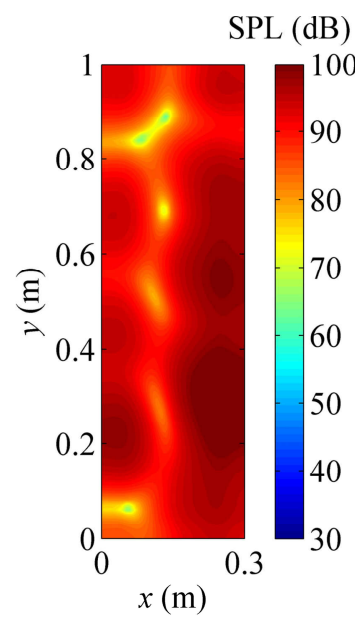

(a)

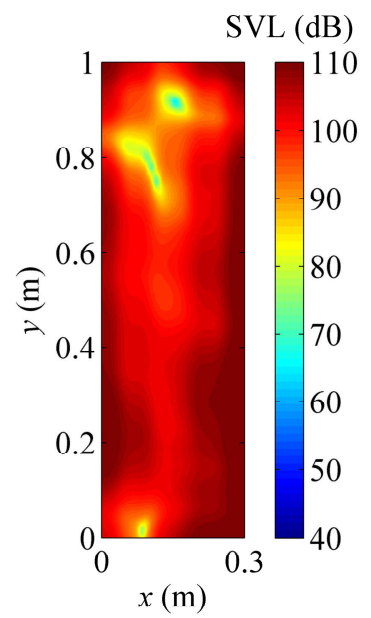

(d)

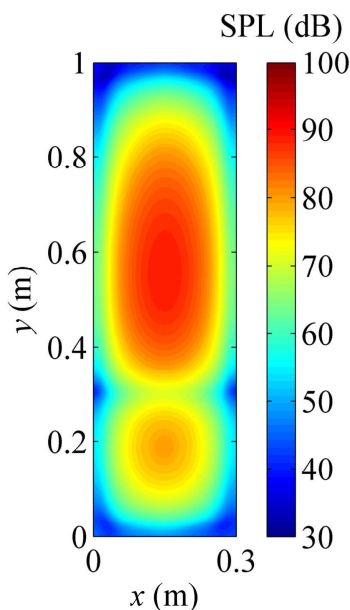

(b)

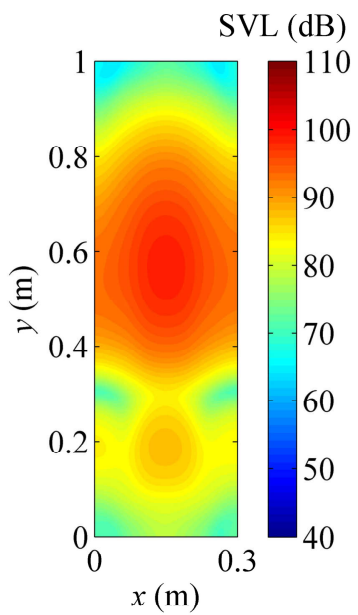

(e)

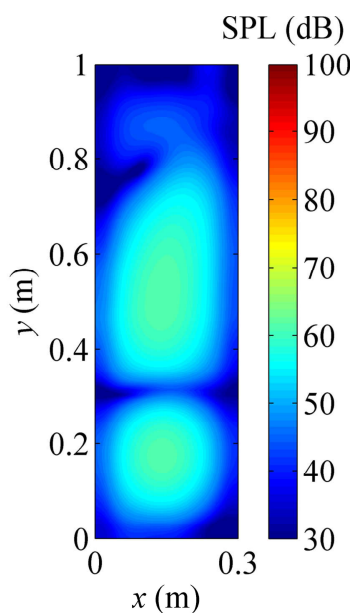

(c)

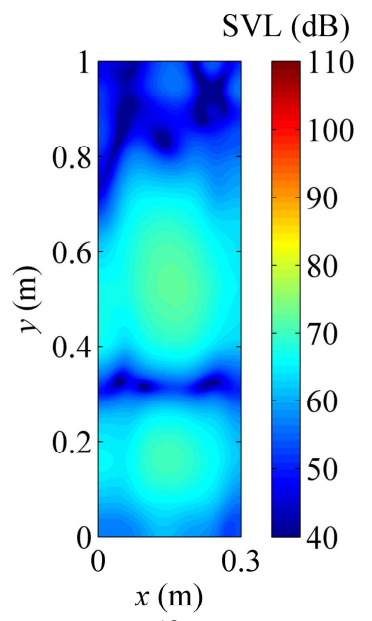

(f)

176 FIG. 4. (Color online) The sound power levels (SPL) at the opening with (a) ANC off; (b) ANC on, single-layer error microphones; and (c) ANC on, double-layer error

178 microphones. The decibel levels of particle velocity (SVL) at the opening with (d) ANC

179 off; (e) ANC on, single-layer error microphones; and (f) ANC on, double-layer error microphones. The frequency of interest is $1000 \mathrm{~Hz}$. In Fig. 3, the noise reductions at $600 \mathrm{~Hz}$ and $1500 \mathrm{~Hz}$ are limited under all the 183 configurations because secondary sources in the same plane cannot excite some of the 
184 modes effectively. ${ }^{26}$ The numerical simulations also show that, unlike using error

185 microphones at the edge, the noise reduction achieved with the system using evenly

186 distributed error microphones can approximate the maximum noise reduction (minimize

187 sound power) if their number is sufficient.

188 It should be noted that double-layer error microphones do not necessarily perform

189 better than single-layer ones. For example, if the secondary source is a monopole point

190 source at $(0.011,0.01,0.01) \mathrm{m}$, which is very close to the primary source, the secondary

191 sound field matches the primary sound field very well, and the noise reduction

192 performances of the single-layer and double-layer error microphones are similar, as

193 shown in Fig. 5. Because strong source coupling exists in this case, the positions of error

194 microphones are not important. In fact, using only one error microphone can achieve

195 similar noise reduction, which is demonstrated by Fig. 5, where the noise reduction

196 performance achieved with one error microphone at $(0.1,0.1,0.588) \mathrm{m}$ is given for

197 comparison. In other cases where the primary and secondary sound fields do not match

198 very well, such as when the secondary source is not located near the primary source, the

199 double-layer error microphones at the edge outperform single-layer ones. In practical

200 applications, there cannot be too many secondary sources, and the number depends on the

201 frequency of the noise to be reduced, but the conclusion that double-layer error

202 microphones outperform single-layer ones is still valid provided the primary and

203 secondary sound fields do not match very well. 


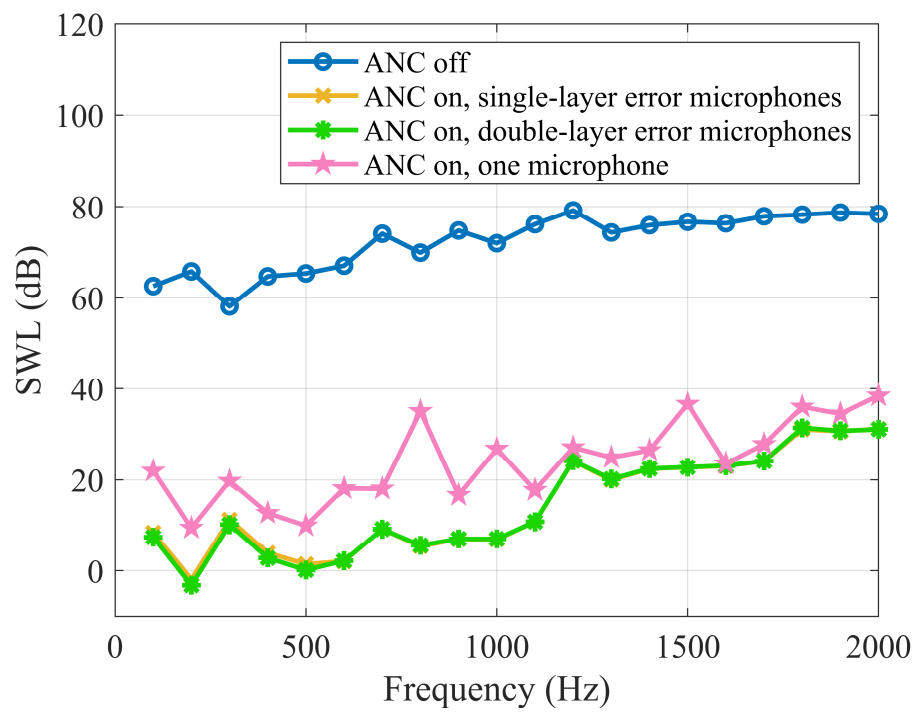

FIG. 5. (Color online) Sound power levels with and without ANC when a single secondary source is located close to the primary source.

\section{B. Upper-limit frequency of effective control}

209 There is a limitation on the control performance of the system with error

210 microphones at the edge. The noise reduction performance achieved with error

211 microphones at the edge will be improved at first if more secondary sources are used, but

212 will remain stable after the number of secondary sources reaches a certain value, and this

213 stable performance is related to the opening size. Using $20 \mathrm{~dB}$ as the threshold, the

214 highest frequency at which the noise reduction is more than $20 \mathrm{~dB}$ with sufficient

215 secondary sources is defined as the upper-limit frequency of effective control.

216 Figure 6 shows the upper-limit frequency as a function of $l_{x}$ when $l_{y}$ and $l_{z}$ are fixed

217 as $1 \mathrm{~m}$ and $0.598 \mathrm{~m}$, respectively. The primary source is located at $(0.01,0.01,0.01) \mathrm{m}$ 
218 and the secondary sources are evenly distributed in the $z=0.448 \mathrm{~m}$ plane. The error

219 microphones in the single-layer system are at the edge of the $z=0.588 \mathrm{~m}$ plane and those

220 in the double-layer system are at the edge of the $z=0.568 \mathrm{~m}$ and $z=0.588 \mathrm{~m}$ planes. In

221 Fig. 6, the upper-limit frequencies of all the systems decrease with $l_{x}$, and the system with

222 double-layer error microphones has higher upper-limit frequencies than that with

223 single-layer error microphones.

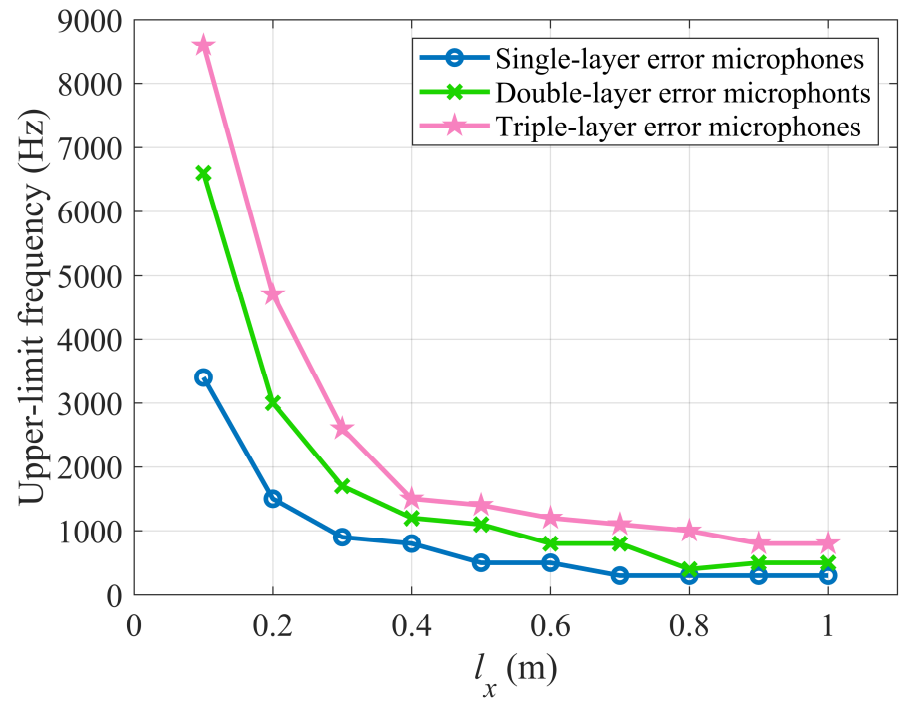

225 FIG. 6. (Color online) Upper-limit frequencies of effective control as a function of $l_{x}$ when the secondary sources are evenly distributed in $z=0.448 \mathrm{~m}$ plane.

229 determined by the smaller side of the opening for a flat opening. For the system with

230 single-layer error microphones, the wavelength of the upper-limit frequency is

231 approximately the length of the smaller side of the cavity opening, while that for the 
232 system with double-layer error microphones is approximately half of this length.

233 Introducing a third layer of error microphones at the edge can further increase the noise

234 reduction achieved by error microphones at the edge. If more error microphone layers are

235 applied, the upper-limit frequency can be improved as well, as shown by the curve

236 corresponding to triple-layer error microphones in Fig. 6.

237 The cavities investigated here are only examples for illustrating the concept and to

238 show that double-layer error microphones at the edge perform better than single-layer

239 ones. Because the upper-limit frequency is related to the size of the opening, such a

240 double-layer error microphone arrangement can be adjusted for applications on openings

241 with different dimensions, and the methodology reported in this paper can be used in

242 other specific designs.

243 If the secondary sound field closely matches the primary sound field, then there is

244 little difference between the performances of using single-layer and double-layer error

245 microphones at the edge. For example, when the secondary source is at $(0.015,0.01,0.01)$

$246 \mathrm{~m}$, which is only $0.005 \mathrm{~m}$ away from the primary source, the upper-limit frequency

247 achieved with a single-layer or double-layer system remains at $3400 \mathrm{~Hz}$ and this

248 frequency does not change with the size of the opening. In this case, strong coupling

249 between the primary and secondary source exists and the upper-limit frequency of

250 effective control is determined by the distance between the primary and secondary

251 sources, so the configuration of error microphones does not have a significant effect on 
252 the noise reduction performance. If the secondary sources cannot be placed in the

253 proximity of the primary source, then the secondary sound field cannot match the primary

254 sound field, and the configuration of error microphones affects the upper-limit frequency.

255 The upper-limit frequencies for more complicated primary sound fields are shown in

256 Fig. 7. The multiple primary sources in the simulations are 27 monopole point sources

257 distributed in a $0.1 \mathrm{~m} \times 0.1 \mathrm{~m} \times 0.1 \mathrm{~m}$ cuboid located from $(0.01,0.01,0.01) \mathrm{m}$ to $(0.11$,

$2580.11,0.11) \mathrm{m}$ with random amplitudes and phases. The results for one primary source at

$259(0.01,0.01,0.01) \mathrm{m}$ are also included in Fig. 7 for comparison.

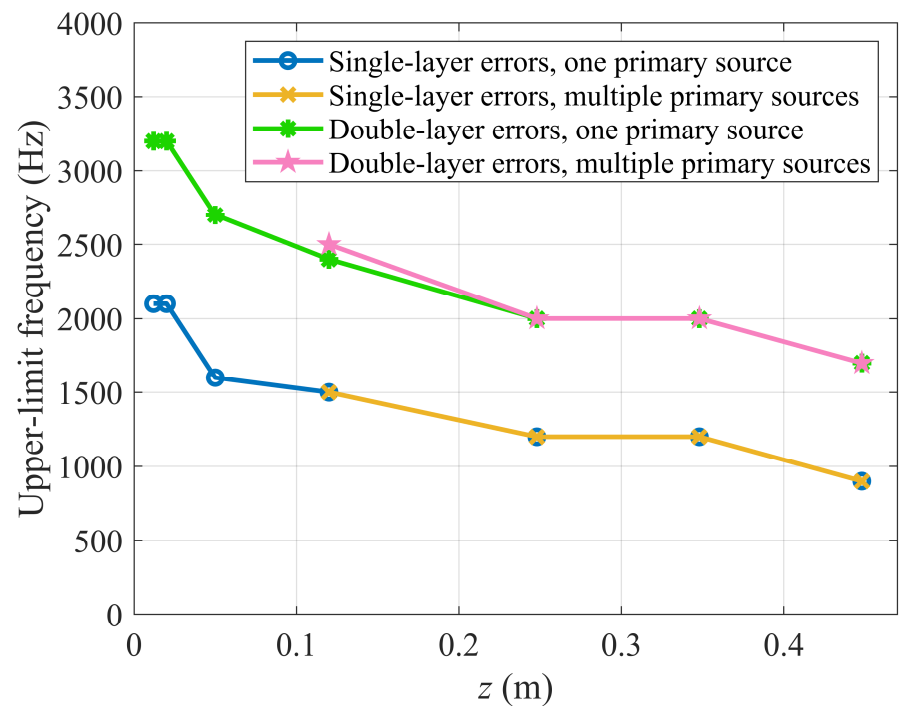

261 FIG. 7. (Color online) Upper-limit frequencies as a function of the plane the secondary sources are located in.

264 It can be seen from Fig. 7 that the upper-limit frequencies for one primary source 265 and multiple primary sources are almost the same, which indicates that the primary sound 
266 field does not affect the upper-limit frequency, but the positions of secondary sources do

267 have an impact on the upper-limit frequencies. As shown in Fig. 7, the upper-limit

268 frequencies decrease with $z$, which is the plane the secondary sources are located in. It

269 indicates that the noise reduction decreases as the secondary sources move farther away

270 from the primary source, and the reason is weaker coupling. In any case, the upper-limit

271 frequency of the system with double-layer error microphones at the edge is always higher

272 than that of the system with single-layer ones.

273 IV. EXPERIMENTS

274 The experiments were carried out in the anechoic chamber of Nanjing University to

275 support the numerical simulation results. A panoramic view of the experimental setup is

276 shown in Fig. 8(a). The cavity size is $0.432 \mathrm{~m} \times 0.67 \mathrm{~m} \times 0.598 \mathrm{~m}$, and the opening is

277 embedded on a baffle $2.4 \mathrm{~m} \times 2.4 \mathrm{~m}$ in size. Ten microphones fixed on a semi-spherical

278 frame with a radius of $1.5 \mathrm{~m}$ were used to measure the sound power levels with and

279 without control according to ISO $3744 .{ }^{11}$

280 In the experiments, 32 secondary sources were evenly distributed in the plane 0.15

$281 \mathrm{~m}$ below the opening and there were 32 error microphones in the system. Three

282 configurations of error microphones: evenly distributed, single-layer and double-layer

283 were investigated and their layouts on the cavity opening are shown in Figs. 8(b)-(d).

284 The single-layer and evenly distributed error microphones were installed in the opening

285 plane. In the double-layer system, two layers of error microphones were installed, one at 
286 the opening and the other one in the plane $0.02 \mathrm{~m}$ below it. A loudspeaker inside the open

287 cavity was used as the primary source to generate a tonal sound field. The waveform

288 synthesis algorithm was used in the experiments; it applied the internally synthesized

289 tonal signal as the reference signal, so no reference microphone is required here. ${ }^{27}$

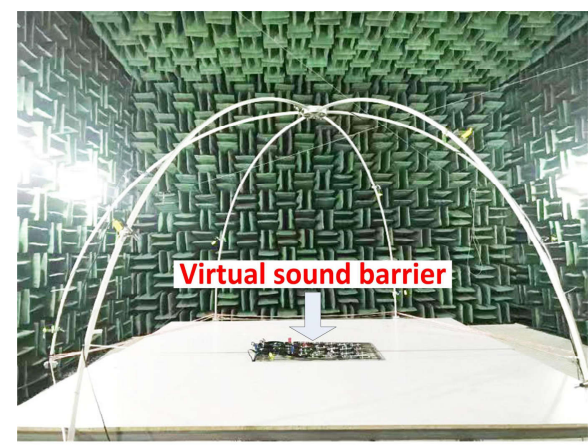

(a)

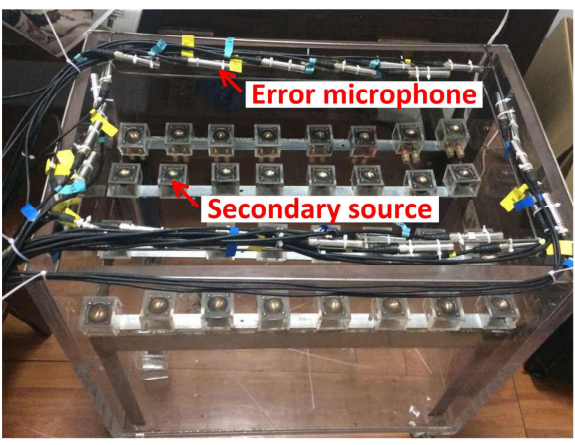

(c)

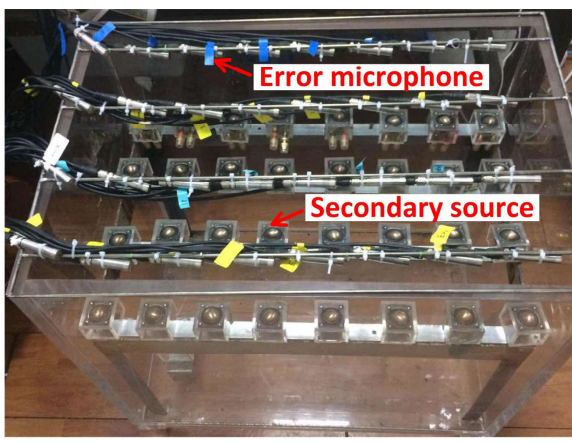

(b)

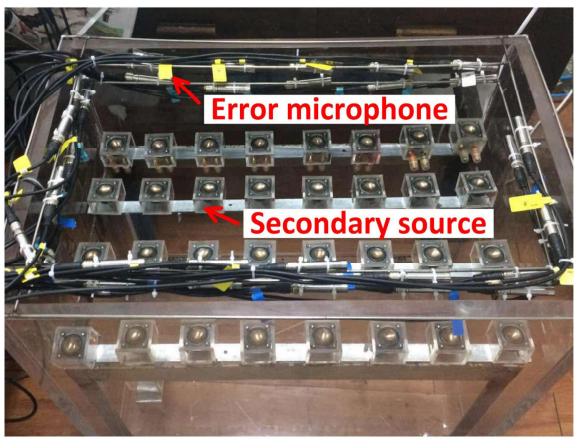

(d)

291 FIG. 8. (Color online) Photos of the experimental setup: (a) a panoramic view of the microphones at the edge, and (d) double-layer error microphones at the edge.

296 shown in Fig. 9(a). It is clear that the system with evenly distributed error microphones

297 has the highest noise reduction among the three configurations. The system with 
299 frequencies between $460 \mathrm{~Hz}$ and $1000 \mathrm{~Hz}$. This is similar to the numerical simulation results shown in Fig. 9(b).
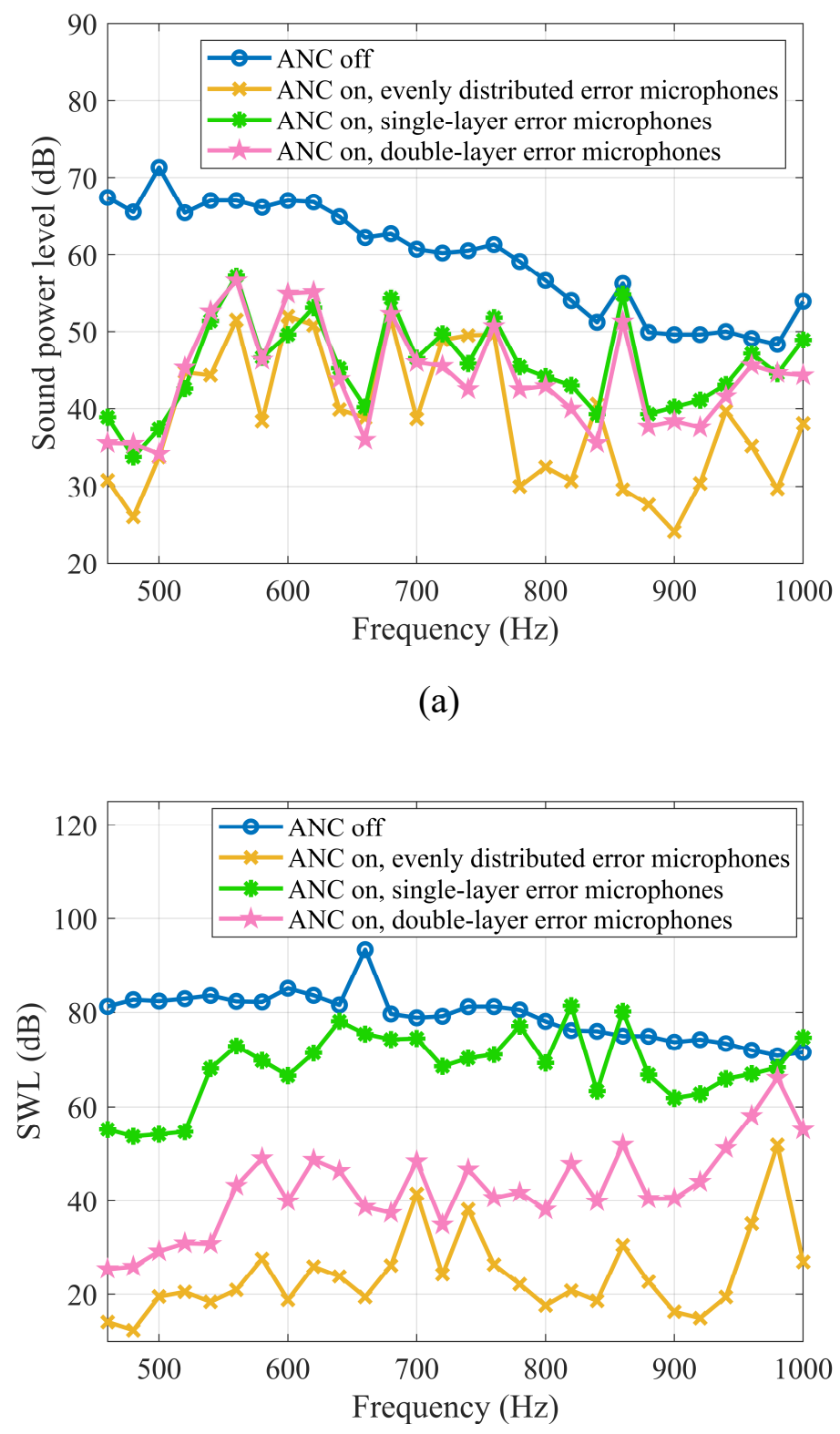

305 FIG. 9. (Color online) (a) Sound power levels with and without ANC measured in the 
308 Unfortunately, the advantage of using double-layer error microphones over using

309 single-layer ones is not as apparent as that in the numerical simulations. There are two

310 possible reasons. One is that the sensitivities of the error microphones are different in the

311 experiments. Because the sum of the squared electric signals picked up by the error

312 microphones was minimized by the active controller, instead of the sum of the squared

313 sound pressures, the noise reduction performance is deteriorated. The other possible

314 reason is that the error microphones in the experiments were not rigorously fixed at their

315 intended positions because of the limited space to install them.

316 Figure 10 shows the numerical simulation results when errors of the microphone

317 sensitivities and positions are considered. The sensitivities of the error microphones used

318 in the experiments ranged from $22.5 \mathrm{mV} / \mathrm{Pa}$ to $39.0 \mathrm{mV} / \mathrm{Pa}$. The maximum error of

319 microphone locations was $1 \mathrm{~cm}$ in each direction from where they were supposed to be. It

320 can be seen from Fig. 10 that with these two factors considered, the difference between

321 the noise reduction achieved with double-layer and single-layer error microphones

322 become less apparent, which demonstrates that these two explanations are reasonable. 


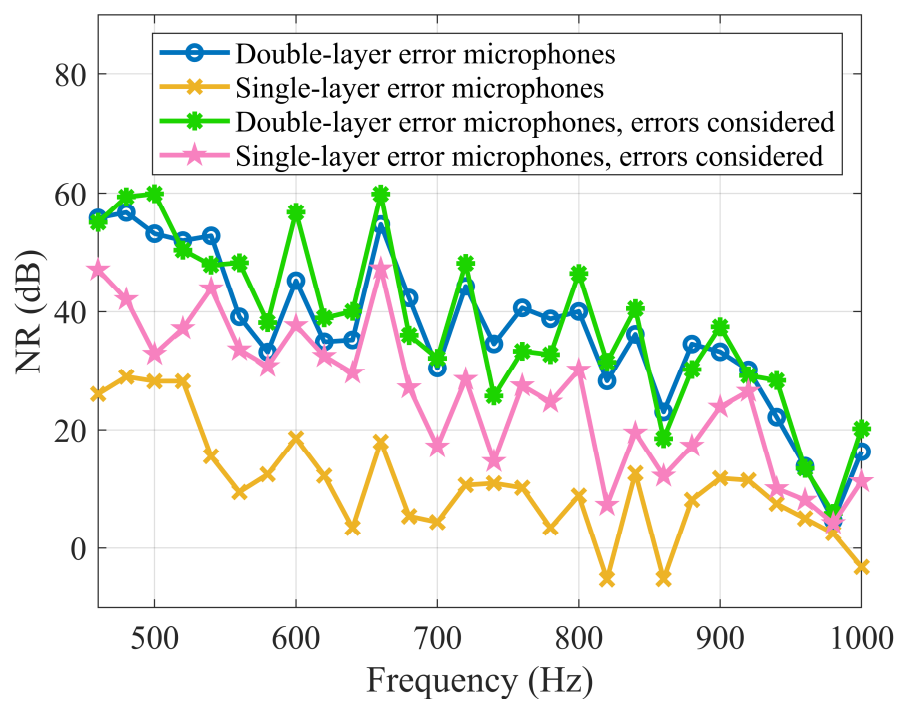

FIG. 10. (Color online) The noise reductions obtained from original numerical

325 simulations and the numerical simulation results with errors of microphone sensitivities

and locations considered.

\section{V. CONCLUSIONS}

329 A boundary error sensing strategy with error microphones at the edge of the cavity

330 opening is proposed to replace the traditional evenly distributed arrangement. It is found

331 that the system with double-layer error microphones at the edge perform better than that

332 with single-layer ones. The reason is that double-layer error microphones enlarge the

333 effective noise reduction zone at the opening. Unlike the system with evenly distributed

334 error microphones, there exists an upper-limit frequency of effective control for the

335 system with error microphones at the edge. Generally, if the secondary sound field cannot

336 match the primary sound field, the upper-limit frequency of effective control is related to 
337 the opening size and more error microphone layers can increase the upper-limit frequency.

338 Experimental results in an anechoic chamber demonstrated the validity of the numerical

339 simulation results. Future work includes combining double-layer secondary sources at the

340 edge with double-layer error microphones at the edge to constitute an almost invisible

341 noise reduction system that has little effect on lighting, natural ventilation, and access

342 through the opening. 


\section{ACKNOWLEDGEMENTS}

344 This research was supported by the National Science Foundation of China (11474163,

345 11874218) and under the Australian Research Council's Linkage Projects funding scheme

346 (LP140100987). The authors would also like to thank Mr. Kang Wang for his help with

347 the experiments.

348 


\section{REFERENCES}

$350 \quad{ }^{1}$ J. Moore and R. Lyon, "Resonant porous material absorbers," J. Acoust. Soc. Am.

$351 \quad$ 72(6), 1989-1999 (1982).

$352{ }^{2}$ J. Kang and M. Brocklesby, "Feasibility of applying micro-perforated absorbers in

353 acoustic window systems,” Appl. Acoust. 66, 669-689 (2005).

$354{ }^{3}$ C. Field and F. Fricke, "Theory and applications of quarter-wave resonators: a prelude

355 to their use for attenuating noise entering buildings through openings," Appl. Acoust.

$356 \quad 53,117-132(1998)$.

$357{ }^{4}$ P. Nelson and S. Elliott, Active Control of Sound, Academic Press, (1992).

3585 S. Ise, "The boundary surface control principle and its applications," IEICE Trans.

359 Fundamentals E88-A, 1656-1664 (2005).

$360{ }^{6}$ M. Nishimura, K. Ohnishi, N. Kanamori and K. Ito, "Basic study on active acoustic

361 shielding," Proceedings of Inter-noise, Shanghai, China (2008).

3627 S. Elliott, J. Cheer, B. Lam, C. Shi and W. Gan, “A wavenumber approach to analysing

363 the active control of plane waves with arrays of secondary sources," J. Sound Vib. 419,

$364 \quad 405-419$ (2018).

365 S. Wang, J. Tao, and X. Qiu, "Performance of a planar virtual sound barrier at the

366 baffled opening of a rectangular cavity,” J. Acoust. Soc. Am. 138(5), 2836-2847

367 (2015). 
3689 S. Wang, J. Tao, and X. Qiu, "Controlling sound radiation through an opening with

369 secondary loudspeakers along its boundaries," Sci. Rep. 7: 13385, 1-6 (2017).

$370{ }^{10} \mathrm{~K}$. Wang, J. Tao and X. Qiu, "Boundary control of sound transmission into a cavity

371 through its opening," J. Sound Vib. 442, 350-365 (2019).

$372{ }^{11}$ ISO 3744: 1994, “Acoustics-Determination of sound power levels of noise sources

373 using sound pressure-Engineering method in an essentially free field over a reflecting

374 plane,” International Organization for Standardization, Geneva, Switzerland, (1994).

$375{ }^{12}$ H. Suzuki, M. Nakamura and J. Tichy, "An accuracy evaluation of the sound power

376 measurement by the use of the sound intensity and the sound pressure methods,"

377 Acoust. Sci. \& Tech. 28(5), 319-327 (2007).

$378{ }^{13}$ A. Berry, X. Qiu, and C. Hansen, "Near-field sensing strategies for the active control of

379 the sound radiated from a plate,” J. Acoust. Soc. Am. 106(6), 3394-3406 (1999).

$380{ }^{14}$ C. Hansen, S. Snyder, X. Qiu, L. Brooks, and D. Moreau, Active Control of Noise and

381 Vibration (CEC Press, Boca Raton, FL, 2012).

$382{ }^{15}$ B. Shafer, K. Gee, and S. Sommerfeldt, "Verification of a near-field error sensor

383 placement in active control of compact noise sources," J. Acoust. Soc. Am. 127,

384 EL66-EL72 (2010).

$385{ }^{16} \mathrm{X}$. Qiu, C. Hansen and X. Li, "A comparison of near-field acoustic error sensing

386 strategies for the active control of harmonic free field sound radiation," J. Sound Vib.

387 215(1), 81-103 (1998). 
$388{ }^{17} \mathrm{~S}$. Rao and T. Pan, "Optimal placement of actuators in actively controlled structures

389 using genetic algorithms,” AIAA Journal. 29(6), 942-943 (1991).

$390{ }^{18} \mathrm{~K}$. Baek and S. Elliott, "Natural algorithms for choosing source locations in active

391 control systems,” J. Sound Vib. 186(2), 245-267 (1995).

$392{ }^{19}$ J. Xue, J. Tao and X. Qiu, "Performance of an active control system near two reflecting 393 surfaces," Proceedings of the 20th International Congress on Sound and Vibration, 394 Bangkok (2013).

$395{ }^{20} \mathrm{~J}$. Garcia-Bonito, S. Elliott and C. Boucher, "Generation of zones of quiet using a 396 virtual microphone arrangement,” J. Acoust. Soc. Am. 101(6), 3498-3526 (1997).

$397{ }^{21}$ A. Berkhoff, "Control strategies for active noise barriers using near-field error sensing,"

398 J. Acoust. Soc. Am. 118(3), 1469-1479 (2005).

$399{ }^{22}$ C. Peterson, A. Zander, B. Cazzolato and C. Hansen, "A moving zone of quiet for 400 narrowband noise in a one-dimensional duct using virtual sensing,” J. Acoust. Soc. Am. $401 \quad$ 121(3), 1459-1470 (2007).

$402{ }^{23}$ C. Fuller, C. Papenfuss and T. Saux, "Active-passive control of portable generator set 403 radiated noise," Proceedings of Acoustics 2012, Fremantle, Australia (2012).

$404 \quad{ }^{24}$ S. Elliott, P. Joseph, P. Nelson and M. Johnson, "Power output minimization and power 405 absorption in the active control of sound," J. Acoust. Soc. Am. 90(5), 2501-2520 406 (1991). 
$407 \quad{ }^{25}$ J. Parkins, S. Sommerfeldt and J. Tichy, "Narrowband and broadband active control in 408 an enclosure using the acoustic energy density,” J. Acoust. Soc. Am. 108(1), 192-203 $409 \quad$ (2000).

$410{ }^{26}$ S. Wang, J. Tao, X. Qiu and J. Pan, "Mechanisms of active control of sound radiation 411 from an opening with boundary installed secondary sources," J. Acoust. Soc. Am. 412 143(6), 3345-3351(2018).

$413{ }^{27} \mathrm{X}$. Qiu and C. Hansen, "An algorithm for active control of transformer noise with 414 on-line cancellation path modelling based on the perturbation method," J. Sound Vib. $415 \quad 240(4), 647-665(2001)$. 


\section{COLLECTED FIGURE CAPTIONS}

419 FIG. 1. (Color online) Schematic diagrams of (a) single-layer error microphones at the

420 edge and (b) double-layer error microphones at the edge.

421 FIG. 2. (Color online) The positions of error microphones in the $x-y$ plane: (a)

422 single-layer error microphones at the edge, (b) one of the layers of the double-layer error

423 microphones at the edge, and (c) evenly distributed error microphones.

424 FIG. 3. (Color online) Sound power levels with and without ANC under different

425 configurations of error microphones compared with the theoretically maximum noise

426 reduction (minimize sound power).

427 FIG. 4. (Color online) The sound power levels (SPL) at the opening with (a) ANC off; (b)

428 ANC on, single-layer error microphones; and (c) ANC on, double-layer error

429 microphones. The decibel levels of particle velocity (SVL) at the opening with (d) ANC

430 off; (e) ANC on, single-layer error microphones; and (f) ANC on, double-layer error

431 microphones. The frequency of interest is $1000 \mathrm{~Hz}$.

432 FIG. 5. (Color online) Sound power levels with and without ANC when a single

433 secondary source is located close to the primary source.

434 FIG. 6. (Color online) Upper-limit frequencies of effective control as a function of $l_{x}$ when

435 the secondary sources are evenly distributed in $z=0.448 \mathrm{~m}$ plane.

436 FIG. 7. (Color online) Upper-limit frequencies as a function of the plane the secondary

437 sources are located in. 
438 FIG. 8. (Color online) Photos of the experimental setup: (a) a panoramic view of the 439 anechoic chamber, (b) evenly distributed error microphones, (c) single-layer error 440 microphones at the edge, and (d) double-layer error microphones at the edge.

441 FIG. 9. (Color online) (a) Sound power levels with and without ANC measured in the 442 experiments. (b) Simulation results on the experimental setup.

443 FIG. 10. (Color online) The noise reductions obtained from original numerical 444 simulations and the numerical simulation results with errors of microphone sensitivities 445 and locations considered. 